\title{
Quantitative Proteomics Reveals Protein Dysregulation During T Cell Activation in Multiple Sclerosis Patients Compared to Healthy Controls
}

\section{Chiara Cappelletti}

Oslo Metropolitan University: OsloMet - storbyuniversitetet

\section{Anna Maria Eriksson}

University of Oslo: Universitetet i Oslo

\section{Ina Skaara Brorson}

Oslo University Hospital: Oslo Universitetssykehus

Ingvild S. Leikfoss

Oslo University Hospital: Oslo Universitetssykehus

\section{Oda Glomstad Kråbøl}

Oslo University Hospital: Oslo Universitetssykehus

\section{Einar August Høgestøl}

Oslo University Hospital: Oslo Universitetssykehus

\section{Valeria Vitelli}

University of Oslo: Universitetet i Oslo

\section{Olav Mjaavatten}

University of Bergen: Universitetet i Bergen

Hanne F. Harbo

Oslo University Hospital: Oslo Universitetssykehus

\section{Frode Steingrimsen Berven}

University of Bergen: Universitetet i Bergen

\section{Steffan D. Bos}

University of Oslo: Universitetet i Oslo

Tone Berge ( $\nabla$ tone.berge@oslomet.no)

OsloMet - storbyuniversitetet https://orcid.org/0000-0002-5297-2034

\section{Research}

Keywords: Autoimmunity, multiple sclerosis, T cell activation, proteomics, disease susceptibility genes

Posted Date: September 9th, 2021 
License: (c) (i) This work is licensed under a Creative Commons Attribution 4.0 International License. Read Full License 
1 Quantitative proteomics reveals protein dysregulation during $\mathrm{T}$ cell activation in multiple sclerosis

\section{2 patients compared to healthy controls}

3 Chiara Cappelletti, ${ }^{1}$ Anna Eriksson, ${ }^{3}$ Ina Skaara Brorson, ${ }^{3,4}$ Ingvild S. Leikfoss, ${ }^{3,4}$ Oda Kråb $\varnothing l,{ }^{2}$ Einar August

4 Høgest $\varnothing \mid,{ }^{3,4,5}$ Valeria Vitelli, ${ }^{6}$ Olav Mjaavatten, ${ }^{7}$ Hanne F. Harbo, ${ }^{3,4}$ Frode Berven, ${ }^{7}$ Steffan D. Bos, ${ }^{4}$ and

$5 \quad$ Tone Berge ${ }^{1,2, *}$

$6 \quad{ }^{1}$ Department of Mechanical, Electronics and Chemical Engineering, Faculty of Technology, Art and

7 Design, OsloMet - Oslo Metropolitan University, Postboks 4, St. Olavs Plass, 0130 Oslo, Norway.

8 2Neuroscience Research Unit, Department of Research, Innovation and Education, Oslo University

9 Hospital, Oslo, Norway

103 Institute of Clinical Medicine, Medical Faculty, University of Oslo, Oslo, Norway.

11 4Department of Neurology, Oslo University Hospital, Ullevål, Postboks 4950, 0424 Nydalen, Oslo,

12 Norway.

$13{ }^{5}$ Department of Psychology, Faculty of Social Sciences, University of Oslo, Oslo, Norway

$14{ }^{6}$ Oslo Centre for Biostatistics and Epidemiology, Department of Biostatistics, University of Oslo, Oslo,

15 Norway.

$16{ }^{7}$ Proteomics Unit at University of Bergen (PROBE), Department of Biomedicine, University of Bergen,

17 Postboks 7804, 5020 Bergen, Norway.

$18 \quad{ }^{*}$ Correspondence: tone.berge@oslomet.no 
Abstract

20 Background: Multiple sclerosis (MS) is an autoimmune, neurodegenerative disorder with a strong 21 genetic component that acts in a complex interaction with environmental factors for disease development. $\mathrm{CD}^{+} \mathrm{T}$ cells are pivotal players in MS pathogenesis, where peripherally activated T cells migrate to the central nervous system leading to demyelination and axonal degeneration. Through a proteomic approach, we aim at identifying dysregulated pathways in activated T cells from MS patients as compared to healthy controls.

Methods: $\mathrm{CD}^{+} \mathrm{T}$ cells were purified from peripheral blood from MS patients and healthy controls by magnetic separation. Cells were left unstimulated or stimulated in vitro through the TCR and costimulatory CD28 receptor for 24 hours prior to sampling. Electrospray liquid chromatographytandem mass spectrometry was used to measure protein abundances.

30 Results: Upon T cell activation the abundance of 1,801 proteins was changed. Among these proteins, 31 we observed an enrichment of proteins expressed by MS-susceptibility genes. When comparing protein abundances in T cell samples from healthy controls and MS patients, 18 and 33 proteins were differentially expressed in unstimulated and stimulated $C D 4^{+} \mathrm{T}$ cells, respectively. Moreover, 353 and 304 proteins were identified as proteins exclusively induced upon T cell activation in healthy controls and MS patients, respectively and dysregulation of the Nur77 pathway was observed only in samples from MS patients.

Conclusions: Our study highlights the importance of $\mathrm{CD}^{+} \mathrm{T}$ cell activation for $\mathrm{MS}$, as proteins that change in abundance upon T cell activation are enriched for proteins encoded by MS susceptibility genes. The results provide evidence for proteomic disturbances in T cell activation in MS, and pinpoint

40 to dysregulation of the Nur77 pathway, a biological pathway known to limit aberrant effector T cell 41 responses.

\section{Keywords}

44 Autoimmunity, multiple sclerosis, T cell activation, proteomics, disease susceptibility genes 
46 Multiple sclerosis (MS) is a complex autoimmune disorder with a significant health and societal burden

$47(1,2)$. It is a chronic inflammatory, demyelinating disorder of the central nervous system (CNS) that 48 leads to both cognitive and physical deficits $(1,3)$. Introduction of disease modifying treatments has 49 ameliorated the conditions of many patients (4), but development of personalized health care is partly 50 precluded due to poor understanding of the biological processes underlying the disease. In addition to 51 major genetic risk variants located in the HLA-gene region, genome-wide association studies (GWAS) 52 have identified additional 200 autosomal MS-associated single nucleotide polymorphisms (SNPs). 53 These findings combined with gene expression profiles have highlighted the importance of several 54 peripheral immune cell types for MS onset, including both the innate and the adaptive immune 55 response (5-7). $C D 4^{+} T$ cells are important regulators of the adaptive immune system and have long 56 been considered to play pivotal roles in MS pathogenesis (8), in which peripheral activation results in 57 migration of these cells into the CNS, leading to demyelination and axonal degeneration (9).

58 Genome-wide studies on epigenetic modifications (e.g. DNA methylation) and gene expression of 59 whole blood, peripheral blood mononuclear cells (PBMCs) and immune cell subtypes have been 60 conducted to investigate potential immune dysregulation in MS. With few exceptions, no overlap was observed between the studies (10-23). Moreover, it is becoming increasingly clear that the correlation between mRNA and protein copy numbers varies widely $(24,25)$, and proteomic studies are therefore needed to complement and confirm findings at the epigenetic or gene expression level. Quantitative

64 high-resolution mass spectrometry-based proteomics enables system-wide studies at the protein level;

65 however, such studies are scarce in samples from individuals with complex diseases such as MS.

66 We have recently performed this approach on $\mathrm{CD}^{+}$and $\mathrm{CD} 8^{+} \mathrm{T}$ cells freshly purified from blood in a 67 small cohort of MS patients and healthy controls (HCS) (26). Importantly, our proteomic study, as well 68 as other studies at the epigenetic and gene expression level, were performed on unstimulated cells and 69 represents an image of the state of the cells at the time of harvesting. Novel disease-associated 
70 pathways could be identified if cells were activated prior to proteomic profiling, as illustrated at the

71 RNA level for MS and coeliac disease, by Hellberg et al. (27) and Quinn et al. (28), respectively.

72 Using liquid chromatography combined with tandem mass spectrometry, we performed quantitative 73 proteomics of $\mathrm{CD}^{+} \mathrm{T}$ cells from relapsing-remitting MS (RRMS) patients and HCs. Cells were left 74 unstimulated or stimulated through the T cell receptor (TCR) in vitro allowing us to disentangle potential $75 \mathrm{CD4}^{+} \mathrm{T}$ cell specific differences induced by $\mathrm{T}$ cell activation, providing novel insights into disease 76 mechanisms of MS. 
MS patients and healthy controls

Blood samples were collected from 20 untreated female RRMS patients (mean age 36.7 years, range 21-63 years) with median extended disability status scale (EDSS) score of 1.5 (range 0-5.5) and mean disease duration of 8 years (range 0.5-38). For one of the patients, the EDSS score was assessed by inspection of their medical journals. HC samples were collected from 20 age- and sex-matched individuals (mean age 37.0 years, range $23-50$ years). All participants were of self-declared Nordic ancestry, and the HCs reported no MS in close family members. MS patients were recruited from the MS out-patient clinic at Oslo University Hospital, Norway, and the HCs from the patients' social networks and among hospital employees. All MS patients fulfilled the updated McDonald criteria for

87 MS at their time of diagnosis (29). At the time of sample collection, the included individuals did not 88 have any ongoing infection, and the MS patients had not experienced a relapse, or received steroids

89 for at least three months prior to enrollment. The Regional Committee for Medical and Health Research

90 Ethics South East, Norway approved the study. Written informed consent was obtained from all study participants.

Isolation of human $\mathrm{CD}^{+}{ }^{+} \mathrm{T}$ cells

Peripheral blood mononuclear cells were isolated from whole blood using density gradient centrifugation with Lymphoprep ${ }^{\mathrm{TM}}$ (Axis Shield, Dundee, Scotland), before negative selection of $\mathrm{CD} 4^{+} \mathrm{T}$ cells with EasySep ${ }^{\text {TM }}$ Human CD4 ${ }^{+}$T Cell Isolation Kit (STEMCELL Technologies, Vancouver, Canada). Cell purity was measured by flow cytometry (Attune Acoustic Focusing Flow Cytometer, Life Technologies, Carlsbad, CA, USA or FACSCalibur, BD Biosciences, Franklin Lakes, NJ, USA) using the fluorescein

99 isothiocyanate-conjugated (FITC) mouse anti-human CD4 antibody (clone RTF-4g) and mouse IgG1 100 isotype control (15H6) (both from Southern Biotech, Birmingham, AL, USA). Aliquots of $\mathrm{CD}^{+} \mathrm{T}^{\mathrm{T}}$ cells 101 were subsequentially frozen with dimethyl sulfoxide (DMSO) (Sigma-Aldrich ${ }^{\circledR}$, Darmstadt, Germany) and stored in liquid nitrogen until usage. 
104 T cell activation

105 Live $\mathrm{CD}^{+}{ }^{+}$T cells stored in liquid nitrogen were thawed and left unstimulated in X-VIVO 15 medium

106 (Lonza, Basel, Switzerland) or stimulated in 96-well plates coated with $5 \mu \mathrm{g} / \mathrm{ml}$ anti-CD3 (mouse anti-

107 human CD3, Clone OKT3, eBioscience ${ }^{\mathrm{TM}}$ by Thermo Fisher Scientific, San Diego, CA, USA) in X-VIVO 15

108 medium supplemented with $2 \mu \mathrm{g} / \mathrm{ml}$ anti-CD28 (purified NA/LE mouse anti-human CD28, BD

109 Biosciences). Cells were cultured at a starting density of 1 million cells $/ \mathrm{ml}$ for $24 \mathrm{~h}$ at $37^{\circ} \mathrm{C}$ and $5 \% \mathrm{CO}_{2}$.

110 Cell pellets of 200000 cells from each sample were kept at $-80^{\circ} \mathrm{C}$ until preparation for mass

111 spectrometry analyses. An aliquot of unstimulated and stimulated CD4 ${ }^{+} \mathrm{T}$ cells were stained with FITC-

112 conjugated mouse anti-human CD69 antibody or mouse IgG1 isotype control (both from ImmunoTools,

113 Friesoythe, Germany) prior to staining with the LIVE/DEAD ${ }^{\text {TM }}$ Fixable Far Red Dead Stain Kit (Invitrogen,

114 by Thermo Fisher Scientific, Carlsbad, CA, USA) for flow cytometry analysis using FACS Canto II flow

115 cytometer (BD Biosciences) to evaluate cell activation and viability. Analysis of flow cytometry data was

116 performed with FCS Express 6 Flow Cytometry Software 2.1 (De Novo Software, Glendale, CA, USA).

117

118 Sample preparation and protein digestion

119 The frozen cell pellets were solubilized in $40 \mu \mathrm{l}$ ice-cold RIPA buffer, containing $1 \% \mathrm{NP} 40,50 \mathrm{mM}$ Tris $\mathrm{HCl}$

$120 \mathrm{pH} 7.6,0.5 \%$ sodium deoxycholate, $0.1 \% \mathrm{SDS}, 150 \mathrm{mM} \mathrm{NaCl}$, and $1 \times$ cOmplete $^{\text {TM }}$ EDTA-free protease

121 inhibitor (cat. No. 11873580001, Roche). Samples were homogenized on ice for 15 min followed by

122 four cycles of ultra-sonification in ice-cold water with $30 \mathrm{sec}$ on and $30 \mathrm{sec}$ off, followed by another 15

123 min on ice. After centrifugation for $10 \mathrm{~min}$ at $16200 \times \mathrm{g}$ at $4^{\circ} \mathrm{C}$, supernatants were collected. Protein

124 concentrations in the lysates were measured by Pierce BCA protein assay (Thermo Fisher Scientific,

125 Rockford, IL, USA) and the absorbance values at $562 \mathrm{~nm}$ were obtained by Multiscan FC 3.1 ELISA reader

126 (Thermo Fisher Scientific, Rockford, IL, USA). Subsequently, $2 \mu \mathrm{l} 150 \mathrm{mM}$ dithiothreitol (DTT) were

127 added to $6 \mu \mathrm{g}$ protein in $25 \mu$ RIPA buffer for cysteine reduction and incubated for 1 hour at RT.

128 Cysteines were alkylated after addition of $3 \mu \mathrm{l} 300 \mathrm{mM}$ iodoacetamide ( 1 hour, at room temperature 
129 protected from light). Digestion of proteins was accomplished using the SP3 protocol (30) with a few

130 modifications: $2 \mu \mathrm{l}(65 \mu \mathrm{g})$ magnetic beads (Sera-Mag SpeedBeads, GE Healthcare, cat. no.

13145152105050250 and 65152105050250) were added to the sample, and the protein

132 binding/aggregation with the beads was accomplished by adding ethanol to $70 \%$ final concentration.

133 After thorough washing in $80 \%$ ethanol, the protein/beads pellet was digested with trypsin (sequencing

134 grade-modified trypsin from Promega, GmbH, Mannheim, Germany) dissolved in $50 \mu \mathrm{l} 100 \mathrm{mM}$

135 ammonium bicarbonate with a trypsin-to-protein ratio of $1: 25$. Samples were incubated at $37^{\circ} \mathrm{C}$ for 16

136 hours at $1000 \mathrm{rpm}$. Tryptic peptides were collected, and beads washed once with $50 \mu \mathrm{l} 0.5 \mathrm{M} \mathrm{NaCl}$.

137 Sample cleanup was performed using a reverse-phase OasisR HLB $\mu$ Elution Plate $30 \mu \mathrm{m}$ (2 mg HLB

138 sorbent, Waters, Milford, MA). After lyophilization, the dried peptides were suspended in $12 \mu$ of 0.5

$139 \%$ formic acid containing $2 \%$ acetonitrile. Two $\mu$ l were used for protein quantification based on

140 absorbance at $280 \mathrm{~nm}$ using a NanoDrop spectrophotometer (Thermo Fisher Scientific, Carlsbad, CA,

141 USA), and $0.6 \mu \mathrm{g}$ of the mixture were analyzed with mass spectrometry.

Liquid chromatography-mass spectrometry/mass spectrometry analysis

144 Peptides were analyzed by electrospray liquid chromatography-tandem mass spectrometry (LC-

$145 \mathrm{MS} / \mathrm{MS}$ ) using a quadrupole-orbitrap instrument (QExactive HF, Thermo Fisher Scientific, Carlsbad, CA,

146 USA). The LC run length of 3 hours was performed on a $50 \mathrm{~cm}$ analytical column (PepMap RSLC, $50 \mathrm{~cm}$

$147 \times 75 \mu \mathrm{m}$ ID EASY-spray column, packed with $2 \mu \mathrm{m}$ C18 beads (Thermo Fisher Scientific, Carlsbad, CA,

148 USA)). Peptides were loaded and desalted on a pre-column (Acclaim PepMap 100, $2 \mathrm{~cm} \times 75 \mu \mathrm{m}$ ID

149 nanoViper column, packed with $3 \mu \mathrm{m}$ C18 beads (Thermo Fisher Scientific, Carlsbad, CA, USA)) with 0.1

$150 \%(v / v)$ trifluoroacetic acid, and eluted with a gradient composition as follows: $5 \%$ B during trapping (5

$151 \mathrm{~min}$ ) followed by $5-8 \% \mathrm{~B}$ for $0.5 \mathrm{~min}, 8-24 \% \mathrm{~B}$ for the next $109.5 \mathrm{~min}, 24-35 \% \mathrm{~B}$ over $25 \mathrm{~min}$, and

$152 \quad 35-80 \%$ B over 15 min. Elution of very hydrophobic peptides and conditioning of the column were

153 performed during $15 \mathrm{~min}$ isocratic elution with $80 \% \mathrm{~B}$ and $20 \mathrm{~min}$ isocratic elution with $5 \% \mathrm{~B}$

154 respectively. Mobile phases $\mathrm{A}$ and $\mathrm{B}$ contained $0.1 \%$ formic acid (vol/vol) in water and $100 \%$ 
155 acetonitrile, respectively, and the flow rate was $200 \mathrm{nl}$ per min. A full scan in the mass area $(\mathrm{m} / \mathrm{z})$ of

$156375-1500$ was performed in the Orbitrap. For each full scan performed at a resolution of $120000(\mathrm{~m} / \mathrm{z}$

157 200), the 12 most intense ions above an intensity threshold of 50000 counts, and charge states 2 to 5

158 were sequentially isolated and fragmented in the Higher-Energy Collision Dissociation (HCD) cell.

159 Fragmentation was performed with a normalized collision energy (NCE) of $28 \%$, and fragments were

160 detected in the Orbitrap at a resolution of $30000(\mathrm{~m} / \mathrm{z} 200)$, with first mass fixed at $\mathrm{m} / \mathrm{z} 100$. One

161 MS/MS spectrum of a precursor mass was allowed before dynamic exclusion for $25 \mathrm{sec}$ with "exclude

162 isotopes" on. Lock-mass internal calibration (m/z 445.12003) was used.

163

164 Mass spectrometry data analysis

165 Mass spectrometry (mass spec) raw files were analyzed by the Proteome Discoverer ${ }^{\mathrm{TM}}$ software

166 (Thermo Fisher Scientific, Carlsbad, CA, USA, version 2.4), and peak lists were searched against the

167 human SwissProt FASTA database (version May 2020), and a common contaminants database by

168 Sequest HT and MS Amanda 2.0 search engines. Methionine oxidation and acetylation on protein N-

169 terminus were added as variable modifications, while cysteine carbamidomethylation was used as fixed

170 modification. False discovery rate (Percolator, http://percolator.ms/) was set to 0.01 for proteins and

171 peptides (minimum length of six amino acids) and was determined by searching the reversed database.

172 Trypsin was set as digestion protease, and a maximum of two missed cleavages were allowed in the

173 database search. Mass recalibration was performed prior to peptide identification using precursor and

174 fragment mass deviation of $20 \mathrm{ppm}$ and 0.5 Da respectively. The main search was then conducted with

175 an allowed mass spec and mass spec/mass spec mass deviation tolerance of $10 \mathrm{ppm}$ and $0.02 \mathrm{Da}$

176 respectively. Retention time alignment and detection of precursor features across samples were done

177 using the Minora Feature Detector node in Proteome Discoverer ${ }^{\mathrm{TM}}$.

178

179 Data processing 
180 A total of 6,687 proteins were identified by the Proteome Discoverer ${ }^{\text {TM }} 2.4$ Software (Thermo Fisher

181 Scientific, Carlsbad, CA, USA). Of these, 178 protein signals were marked as contaminants and therefore

182 removed from further analysis. In Perseus (Perseus Software, version 1.5.6.0), the normalized

183 abundances from Proteome Discoverer ${ }^{\mathrm{TM}}$ were log2 transformed and the normal distributions were

184 controlled by plotting the histograms. Proteins with valid values in at least $70 \%$ of the samples in at

185 least one of the four groups (HC: unstimulated, HC: stimulated, MS: unstimulated and MS: stimulated)

186 were used for analysis. The missing protein abundances were imputed from the normal distribution

187 using default settings in Perseus.

188

189 Statistical analyses

190 All analyses presented were performed using the R software version 4.0.4. Differences in protein

191 abundances upon T cell activation were assessed using a paired two-tailed Student's $t$-test. When

192 comparing protein abundance between samples from MS patients and HCs, a Welch's test (for unequal

193 variances) was used. Principal component analysis (PCA) plots were generated using protein intensities

194 of differentially expressed proteins as variables. For validation analysis, 100 discovery cohorts were

195 simulated by randomly selecting ten MS samples and ten HC samples and the differentially expressed

196 proteins identified in these simulated cohorts were used as input for performing PCA in the remaining

197 samples.

198 Within each analysis stratum, the Benjamini-Hochberg (B-H) procedure was used to correct for multiple

199 testing and adjusted p-values considered significant are indicated in the results section.

200

201 Ingenuity pathway analysis

202 QIAGEN’s Ingenuity ${ }^{\circledR}$ pathway Analysis (IPA ${ }^{\circledR}$ QIAGEN, version 52912811, date: 2020-09-07) was used

203 for functional interpretation of significantly expressed proteins. The default settings were used, species

204 was set to "all" and "T lymphocytes", "Immune cell lines", "CCRF-CEM", "Jurkat" and "MOLT-4" were 

used, and a value below 0.05 (-log (B-H p-value) > 1.3) was considered significant. 
209 In this study, we examined the differences at the proteomic level of $\mathrm{CD}^{+} \mathrm{T}$ cells from RRMS patients

$210(n=20)$ and HCs $(n=20) . C D^{+} T$ cells were left unstimulated or stimulated through the TCR (anti-CD3;

211 OKT3) and costimulatory CD28 receptor (anti-CD28) for 24 hours prior to sampling (Figure 1.A). T cell

212 activation was verified by measuring the cell surface expression of the T cell activation marker CD69 by

213 flow cytometry (Figure 2.A). Using a label-free proteomics approach, we were able to identify and

214 quantify a total of 5,704 proteins. Of these proteins, the abundance of 1,801 was changed upon T cell

215 activation (adjusted $\mathrm{p} \leq$ 0.01) (Figure 1.B).

216 When comparing protein abundances in the T cell samples from HCs and MS patients, 18 and 33

217 proteins were differentially expressed (adjusted $p \leq 0.05$ ) in unstimulated (Table 1, Figure 1.C) and

218 stimulated $C D 4^{+}$T cells (Table 2, Figure 1.D), respectively, with two proteins; diphthamide synthetase,

219 encoded by $D P H 6$, and enhancer of polycomb homolog 1, encoded by EPC1, being significant in both conditions. Diphthamide synthetase expression was higher in unstimulated cells from MS patients (log2 fold change $=3.30$ ), whereas its expression was lower in stimulated cells from MS patients (log2 fold change $=-1.91)$, compared to HC. Enhancer of polycomb homolog 1 showed higher fold change between MS and $\mathrm{HC}$ samples in stimulated samples (log2 fold change $=3.47)$ compared to unstimulated samples (log2 fold change $=2.34)$. The principal component analysis $(P C A)$ plots of significant proteins in each analysis show separated clusters of samples from MS patients and HCs in unstimulated (Figure 3.A) and stimulated (Figure 3.B) $\mathrm{CD}^{+} \mathrm{T}$ cells. Close to $50 \%$ of the total variation in the dataset, which captures the separation between MS and HCs, was explained by the first component, whereas the second component captured $11-12 \%$ of the variance in each analysis.

Validation of protein dysregulation in $\mathrm{CD}^{+} T$ cells from MS patients by resampling

231 To validate the protein dysregulation observed in $\mathrm{CD}^{+} \mathrm{T}$ cells from MS patients, we simulated 100

232 discovery cohorts by randomly selecting ten MS samples and ten HC samples $\left(\mathrm{n}_{\mathrm{MS}}=10, \mathrm{n}_{H C}=10\right)$ for 
233 each simulated dataset. For both conditions (unstimulated and stimulated), we performed differential

234 expression analysis in each of the 100 simulated discovery cohorts. We carried out PCA analysis based

235 on the differentially expressed proteins (adjusted $p \leq 0.05$ ) in each corresponding replication cohorts,

236 consisting of the remaining samples $\left(n_{M S}=10, n_{H C}=10\right)$. The number of significant proteins in the main

237 analysis and the median number of significant proteins obtained from the validation analysis for each

238 condition are listed in Table 3. The lower number of significant proteins found in the validation analysis

239 is due to the lower power to detect differentially expressed proteins in smaller datasets ( $n=10$ versus

$240 \mathrm{n}=20$ ). In the validation analysis, we found that the scores of the first principal components were

241 statistically different ( $p \leq 0.05$ ) between $\mathrm{MS}$ and $\mathrm{HC}$ samples in $82 \%$ of the iterations for the

242 unstimulated samples and in $61 \%$ of the iterations in the stimulated samples. Of note, in two out of

243100 iterations in the unstimulated samples, no significant proteins were found, whereas in the

244 validation analysis of the stimulated samples, significant proteins were found in all iterations. These

245 analyses confirmed that most of the variance present in our samples captured by the first principal

246 component was due to protein dysregulation in $\mathrm{CD}^{+} \mathrm{T}$ cells between MS patients and HCs.

247 When comparing the differentially expressed proteins in samples from MS patients and HCs identified

248 in the iteration analyses, we discovered that diphthamide synthetase, encoded by DPH6, was found in

24998 iterations of the unstimulated samples, while Grb2-related adapter protein and enhancer of

250 polycomb homolog, encoded by GRAP and EPC1, respectively, were found in all 100 iterations from

251 stimulated samples.

252

253 Proteins differentially expressed upon T cell activation are enriched for proteins expressed by MS-

254 susceptibility genes

255 To test for enrichment of proteins encoded by MS susceptibility genes among the 1,801 proteins whose

256 abundance is changed upon T cell activation (Figure 1.B), the IDs of 285 most proximal genes were

257 extracted from the list of 200 autosomal, non-HLA MS-associated SNPs (7). For intergenic MSassociated SNPs, we extracted the most proximal genes both upstream and downstream of the SNPs. 
259 Out of these, 34 gene IDs corresponded to non-coding RNAs and were removed from the analysis. Not

260 all MS susceptibility genes are expressed in T cells, and in our samples, we detected 97 proteins

261 encoded by MS susceptibility genes that were expressed either in the unstimulated or stimulated samples. Of these, 43 proteins were among the 1,801 differentially expressed upon T cell activation regardless of the disease status. A Person's Chi-squared test showed that there was a significant enrichment for proteins encoded by MS susceptibility genes among the 1,801 proteins that were changed upon T cell activation ( $p=0.0089$; Table 4), highlighting the importance of this process in MS.

269 To elaborate on the differences in the T cell activation process in $C D 4^{+} \mathrm{T}$ cells from MS patients and

270 HCs, we specifically analyzed proteins that displayed a significant change in abundance upon $\mathrm{T}$ cell activation in HC and MS (Figure 1.E). We discovered 990 differentially expressed proteins (adjusted $p \leq$ 0.01) between unstimulated and stimulated $\mathrm{CD}^{+} \mathrm{T}$ cells in $\mathrm{HCs}$ and 941 differentially expressed proteins in MS patients. Of these proteins, 637 were differentially expressed in both HC and MS samples, whereas 353 and 304 proteins were exclusively differentially expressed upon $\mathrm{CD}^{+}{ }^{+}$cell activation in HCS and in MS patients, respectively (Figure 1.E). Of the 637 proteins differentially expressed in both groups, all proteins, except for pyruvate dehydrogenase and Late genes, showed a change in expression in the same direction across the groups.

279 The IPA software was used for network analyses of proteins whose expression was affected by $T$ cell

280 activation exclusively in samples from MS patients or HCs. We identified enrichment in ten biological

281 processes (Figure 4.A; $-\log (\mathrm{B}-\mathrm{H}$ p-value $)>1.3$ ) among the proteins exclusively changed upon stimulation

282 of $\mathrm{CD}^{+} \mathrm{T}$ cells from MS patients, whereas among the proteins exclusively changed upon activation of

$283 \mathrm{CD}^{+} \mathrm{T}$ cells from $\mathrm{HCs}$, we identified one biological process (Figure 4.B; - $\log (\mathrm{B}-\mathrm{H}$ p-value) $>1.3$ ). The top

284 four pathways (elF2 signaling, regulation of elF4 and p70S6K signaling, Coronavirus pathogenesis 
285 pathway, and mTOR signaling) identified among the proteins exclusively changed in MS patients

286 corresponded to the top four pathways identified among the proteins whose expression were changed

287 upon T cell activation in both groups (Table 5). Of note, the Nur77 signaling pathway identified among

288 the proteins exclusively changed upon activation of $\mathrm{CD}^{+} \mathrm{T}$ cells from HCs has been shown to be a key

289 regulator of T cell function by restricting activation, cell cycle progression, and proliferation (31). 
291 Genome-wide association studies have revealed 230 risk loci for MS, mostly located within or close to

292 genes expressed in immune cells (7). However, it remains to be analyzed whether genetic differences

293 are translated into cell-specific expression profiles in samples from MS patients and HCs. Previous

294 transcriptomic analyses of $\mathrm{CD}_{1} 4^{+}$monocytes, $\mathrm{CD} 4^{+}$and $\mathrm{CD} 8^{+} \mathrm{T}$ cells, indicated that $\mathrm{CD} 4^{+} \mathrm{T}$ cells were

295 the most dysregulated cell type in MS among these three immune cells (32). Transcriptomic profiling is

296 frequently performed to identify genes and pathways of relevance for complex diseases such as MS.

297 Given the lack of complete correlation between mRNA and protein copy numbers (24, 25), proteomic

298 profiling enables an alternative or complementary approach for identification of disease relevant

299 pathways. To our knowledge, we were the first to perform proteomic profiling of purified immune-cell

300 subsets from MS patients. Using electrospray liquid chromatography-tandem mass spectrometry, we

301 were able to identify aberrant protein expression in freshly purified T cells, i.e. $C D 4^{+}$and $C D 8^{+} T$ cells,

302 from MS patients as compared to HCs (26). In the current study, we used the same technique for

303 proteomic profiling of $\mathrm{CD}^{+} \mathrm{T}$ cell samples left unstimulated or stimulated for 24 hours in vitro through

304 the TCR, to analyze protein dysregulation during T cell activation in MS. We identified novel proteins

305 that were differentially expressed in response to activation in samples from MS patients as compared

306 to HCs. Furthermore, we found that the proteins, whose expression was changed upon T cell activation,

307 were enriched for proteins encoded by MS susceptibility genes. These findings confirmed the

308 importance of $\mathrm{CD}^{+} \mathrm{T}$ cell activation for MS pathogenesis. As the included patients already had

309 developed MS, it remains to be shown whether this aberrant response contributes to developing MS

310 or rather is a consequence of the ongoing disease. Of note, all included MS patients were untreated

311 and clinically stable at the time of sample collection, excluding the possibility for disease modifying

312 treatment having affected the T cells used in this study.

313 There is little overlap between the findings from this study and Berge et al., 2019 (26), but both studies

314 were relatively low powered due to the small sample sizes. To rule out findings attributable to low

315 sample size, a validation analysis was performed in the current study and confirmed the protein 
316 dysregulation observed in MS patients. Furthermore, even though eight samples (four MS and four

317 HCs) were obtained from the same donors as included in (26), the experimental set ups were different

318 between the two studies. In our previous study (26), the $\mathrm{CD}^{+} \mathrm{T}$ cells were prepared for mass

319 spectrometry directly after cell purification to investigate their status in MS patients. On the contrary,

320 for this study, live cells were stored on liquid nitrogen prior to thawing and cell cultivation for 24 hours

321 in the presence or absence of stimulating antibodies to investigate T cell behavior upon activation. All

322 samples included in this study were treated equally, and there was no difference between the two

323 groups in cell viability or cell activation (Figure 2), as measured by cell surface expression of CD69 using

324 flow cytometry. Using our stimulation protocol, cells were triggered through the TCR and CD28 co-

325 receptor. Therefore, all T cells in the culture, independent of specificity and binding strength, were

326 likely to be activated, ruling out the possibility of a different TCR repertoire in the MS population.

327 Through proteomic profiling of stimulated cells, we identified MS-associated proteins that were

328 hitherto not identified with the current available approaches, i.e. global DNA methylation analyses or

329 RNA sequencing, performed in untreated immune cell subsets, full blood or in PBMCs (10-23).

330

331 There were only two proteins differentially expressed between MS patients and HCs in both the unstimulated and stimulated samples, i.e. diphthine:ammonia ligase (also called diphthamide synthetase) encoded by DPH6 and enhancer of polycomb homolog 1 encoded by EPC1. Diphthamide synthetase catalyzes the conversion of histidine to diphthamide for regulation of the translation factor

335 EEF2 (33), which controls neurological processes (34), but with hitherto no known role in autoimmunity.

336 Enhancer of polycomb homolog 1 is a transcriptional regulator (35) with no known function in T cells

337 and was also one of two proteins differentially expressed in all the 100 iterations performed with the

338 stimulated samples. Another protein differentially expressed in all the 100 iterations and the top hit of

339 the main analysis in the stimulated samples (log2 fold change $=5.35)$, was Grb2-related adapter protein

340 encoded by GRAP. Of note, Grb2-related adapter protein 2 encoded by the MS susceptibility gene 341 GRAP2 was expressed at higher levels in $\mathrm{CD}^{+} \mathrm{T}$ cells from MS patients as compared to HCs in our 
342 previously published proteomic analyses (26). The Grb2 family of adapter proteins has been shown to

343 interact with the activated T cell costimulatory receptor CD28 (36) and to be involved in Erk-MAP kinase

344 activation in human B cells (37). Moreover, the GRAP gene is primarily expressed in human thymus and

345 spleen (38), and it negatively regulates TCR-elicited proliferation and interleukin-2 induction in murine

346 Iymphocytes (39). Identification of these adapters in our proteomic approaches suggests further

347 investigation of the Grb2 family of adaptor proteins in MS.

348

349 Among the differentially expressed proteins between MS patients and HCs, three proteins have 350 previously been suggested to play a role in MS pathogenesis: (1) tyrosine kinase 2 (TYK2), (2) protein 351 tyrosine phosphatase non-receptor type 2 (PTPN2), and (3) DNA polymerase subunit gamma-1 (POLG).

352 In our data set, TYK2 was slightly upregulated in unstimulated samples from MS patients (log2 fold 353 change $=1.14)$. An exonic TYK2 variant ( $r$ 34536443) has been shown to associate with increased MS 354 risk (7), and the presence of the protective allele at rs3453443 resulted in reduced TYK2 kinase activity 355 in T cells and a shift in the cytokine secretion profile favoring Th2 development, but did not modify TYK2 expression when measured by Western blotting (40). With a minor allele frequency of 0.01423 (www.snpedia.com) for the MS associated rs34536443 SNP in TYK2 and the limited sample size in the presented study, it is unlikely that the genotype of this SNP underlies the difference in TYK2 expression between the two groups. PTPN2 has previously been linked to MS as a microRNA, i.e. miR-448, that was upregulated in PBMC and cerebrospinal fluid (CSF) from MS patients, promoted IL-17 production

361 directly through PTPN2, thereby contributing to development of an autoinflammatory immune 362 environment. However, being a direct target of miR-448, PTPN2 expression was reduced in PBMC and 363 CSF from MS patients (41), whereas we observed a small increase in stimulated $\mathrm{CD} 4^{+} \mathrm{T}$ cells from MS

364 patients (log2 fold change $=0.85)$. Nevertheless, the experimental set-up and the biological materials 365 were different in the two studies. In our analyses, we were able to detect cell-specific differences, which 366 could be convoluted when analyzing heterogeneous samples such as PBMCs or CSF. POLG expression 367 was reduced in stimulated $\mathrm{CD}^{+}{ }^{+} \mathrm{T}$ cells from MS patients (log2 fold change $=-1.35$ ) as compared to $\mathrm{HC}$ 
samples. Genetic variants in the POLG gene have been associated with familiar MS (42). In a smaller genetic study, POLG was suggested as an MS susceptibility gene (43), but it did not reach genome-wide significance in the latest MS GWAS (7).

371

372 As MS is an autoimmune disease, it is not a surprise that proteins expressed from MS susceptibility 373 genes are enriched among the proteins that change expression upon T cell activation, highlighting the 374 importance of this process in MS. Findings from our previous proteomic study (26) also pointed to the 375 importance of $\mathrm{T}$ cell activation, as the differentially expressed proteins between $\mathrm{CD} 4^{+} \mathrm{T}$ cells from MS 376 patients and HCs were enriched in pathways related to T cell activation. In the current study, most 377 proteins that were induced or inhibited upon $\mathrm{CD}^{+} \mathrm{T}$ cell stimulation were overlapping in samples from 378 MS patients and HCs. However, there were sets of proteins that were selectively regulated in one group 379 only. Pathway analyses showed that proteins with changes in expression upon T cell activation in the

380 MS group only correspond to pathways also identified among the proteins changed upon $T$ cell 381 activation in both groups, including pathways of translation initiation and immune response (elF2 and 382 elF4) and cell survival and proliferation (mTOR). Interestingly, pathway analysis showed that proteins 383 with changes in expression upon T cell activation in the HC group only were enriched for the Nur77 384 pathway. This signaling pathway limits aberrant effector T cell responses and impedes the development 385 of T cell-mediated inflammatory diseases such as autoimmune disorders (31). Our data suggest that 386 the Nur77 pathway is not induced upon T cell activation in MS patients as in HCs, and more thorough 387 functional analyses are needed to understand its role for development of MS.

388 In the current study, we examined one immune cell subtype from blood, CD4 ${ }^{+} \mathrm{T}$ cells, which provided 389 a detailed insight into one specific immune cell subtype with a likely role in MS. However, it should be 390 noted that $C D 4^{+} T$ cells can be further divided into subclasses and consequently differences in subtypes 391 of $\mathrm{CD}^{+} \mathrm{T}$ cells, such as Th17 or regulatory $\mathrm{T}$ cells, might not be detected, as these signals may be 392 concealed by signals from the more abundant $C D 4^{+} T$ cells subtypes. Although we have identified novel 
393 proteins of potential importance for MS, further studies are needed to validate and verify the biological

394 impact of selected proteins and pathways in T cells.

395

396 Conclusions

397 In summary, using electrospray liquid chromatography-tandem mass spectrometry for analyses of in

398 vitro stimulated $\mathrm{CD}^{+} \mathrm{T}$ cells from MS patients and $\mathrm{HCs}$, we were able to identify aberrant regulation of 399 novel proteins in MS, hitherto not identified through other approaches. Proteins encoded by MS

400 susceptibility genes are enriched among proteins that change in abundance upon T cell activation, and

401 through pathway analyses, we have identified enrichment of induced proteins from the Nur77 pathway

402 in HC samples only.

403

404 List of abbreviations

405 MS: Multiple sclerosis

406 HC: Healthy control

407 CNS: Central nervous system

408 GWAS: Genome wide association study

409 SNP: Single nucleotide polymorphism

410 PBMC: Peripheral blood mononuclear cell

411 RRMS: Relapsing-remitting multiple sclerosis

412 TCR: T cell receptor

413 EDSS: Extended disability status scale

414 FITC: Fluorescein isothiocyanate-conjugated

415 DMSO: Dimethyl sulfoxide

416 DTT: Dithiothreitol

417 LC-MS/MS: Electrospray liquid chromatography-tandem mass spectrometry

418 HCD: Higher-energy collision dissociation 
419 NCE: Normalized collision energy

420 Mass spec: Mass spectrometry

421 PCA: Principal component analysis

422 B-H: Benjamini-Hochberg

423 IPA: Ingenuity pathway analysis

424 TYK2: Tyrosine kinase 2

425 PTPN2: protein tyrosine phosphatase non-receptor 2

426 POLG: DNA polymerase subunit gamma-1

427 CSF: Cerebral spinal fluid

428

429 Declarations

430 Ethics approval and consent to participate

431 The Regional Committee for Medical and Health Research Ethics South East, Norway approved the

432 study. Written informed consent was obtained from all study participants.

433

434 Consent for publication

435 Not applicable.

436

437 Availability of data and materials

438 The datasets generated in the study are currently being uploaded into the PRoteomics IDEntifications

439 (PRIDE) database and will be publically available.

440

441 Competing interests

442 TB received unrestricted research grants from Biogen Idec and Sanofi-Genzyme, EAH received

443 honoraria for lecturing and advisory board activity from Biogen, Merck and Sanofi-Genzyme and

444 unrestricted research grant from Merck, ISL received unrestricted research grant from Novartis and 
445 HFH has received travel support, honoraria for advice or lecturing from Biogen, Merck, Sanofi-

446 Genzyme, Roche and an unrestricted research grant form Merck. The other authors declare that they 447 have no competing interests.

448

449 Funding

450 This research was supported by grants from the South-Eastern Health Authorities of Norway [2017114];

451 the Research Council of Norway [240102]; Oslo Metropolitan University; and unrestricted research

452 grants from Biogen Idec, Sanofi-Genzyme, Odd Fellow and Halvor Høies Fund. Mass spectrometry-

453 based proteomic analyses were performed by The proteomics Unit at University of Bergen (PROBE).

454 This facility is a member of the National Network of Advanced Proteomics Infrastructure (NAPI), which

455 is funded by the Research Council of Norway INFRASTRUKTUR-program (project number: 295910).

456

457 Authors' contributions

458 TB and AE conceived the idea. CC, TB and FB planned the study. AE, ISB, ISL, EAH, HFH, SDB and TB

459 recruited MS patients and healthy controls. CC, AE, ISB, ISL, OK, SDB and TB collected samples. OM

460 carried out mass spectrometry. CC, AE, ISB, OK, VV, SDB, OM, FB and TB analyzed and interpreted the

461 data. CC and TB wrote the manuscript. All authors revised the manuscript and approved the final

462 manuscript.

463

464 Acknowledgements

465 We thank all patients and healthy controls for participation, Elisabeth G. Celius and Marte Wendel-Haga

466 for patient recruitment, Anja Bjølgerud for technical assistance and research nurses for drawing blood

467 samples.

468 
471 Figure 1. An overview of the study. Study design (A). Differentially expressed proteins between unstimulated and 472 stimulated $C D 4^{+}$T cells (B). Differentially expressed proteins between $\mathrm{HC}$ and MS in unstimulated $\mathrm{CD} 4^{+} \mathrm{T}$ cells (C) 473 and in stimulated $C D 4^{+} T$ cells (D). Proteins that change in abundance upon $\mathrm{CD}^{+} \mathrm{T}$ cell activation of samples from

$474 \mathrm{MS}$ and $\mathrm{HC}(\mathrm{E})$. The Venn diagram displays the number of proteins that were differentially expressed between 475 unstimulated and stimulated $\mathrm{CD}^{+} \mathrm{T}$ cells from HCs (blue) and MS patients (red).

Figure 2. Flow cytometry characterization of $\mathrm{CD4}^{+} \mathrm{T}$ cells from MS patients (MS) and healthy controls (HC).

478 Proportions of (A) $\mathrm{CD} 9^{+}$and (B) viable $\mathrm{CD}^{+} \mathrm{T}$ cells in unstimulated and stimulated (anti-CD3/CD28 antibody 479 stimulation) samples. Mean and standard deviation are shown, Mann Whitney $U$ test was used for paired 480 comparisons, only significant $p$-values $(p>0.05)$ are depicted in the figure. $M S=$ multiple sclerosis, $H C=$ healthy 481 controls.

482

483 Figure 3. Principal component analysis (PCA) of differentially expressed proteins. Scores of the first (PC1) and 484 second (PC2) principal components obtained by PCA of proteins significantly different in abundance ( $p \leq 0.05)$ 485 between MS patients (MS; red circles) and HCs (HC; blue triangles) in (A) unstimulated and (B) stimulated CD4 ${ }^{+}$ 486 cells.

488 Figure 4. Biological pathways enriched upon $\mathrm{CD}^{+} \mathrm{T}$ cell activation. The graphs display the biological pathways 489 enriched among proteins that are differentially expressed between unstimulated and stimulated CD4 ${ }^{+} \mathrm{T}$ cells 490 exclusively in (A) MS patients (pink) or (B) HCs (light blue) after Benjamini-Hochberg (B-H) multiple testing 491 correction ( $p$-values seen on left y axis, blue line is marking the threshold level for significance). The red line with 492 squares represents the ratio of the number of proteins in the data set of differentially expressed proteins divided 493 by the number of proteins in the reference data set for that specific pathway (right y axis). 
495 1. Thompson AJ, Baranzini SE, Geurts J, Hemmer B, Ciccarelli O. Multiple sclerosis. Lancet. 496 2018;391(10130):1622-36.

$4972 . \quad$ Collaborators GBDN. Global, regional, and national burden of neurological disorders, 1990498 2016: a systematic analysis for the Global Burden of Disease Study 2016. Lancet Neurol. 499 2019;18(5):459-80.

$500 \quad 3 . \quad$ Dendrou CA, Fugger L, Friese MA. Immunopathology of multiple sclerosis. Nat Rev Immunol. $501 \quad 2015 ; 15(9): 545-58$.

502 4. Tintore M, Vidal-Jordana A, Sastre-Garriga J. Treatment of multiple sclerosis - success from bench to bedside. Nat Rev Neurol. 2019;15(1):53-8.

504 5. International Multiple Sclerosis Genetics C, Wellcome Trust Case Control C, Sawcer S, 505 Hellenthal G, Pirinen M, Spencer CC, et al. Genetic risk and a primary role for cell-mediated immune 506 mechanisms in multiple sclerosis. Nature. 2011;476(7359):214-9.

507 6. International Multiple Sclerosis Genetics C, Beecham AH, Patsopoulos NA, Xifara DK, Davis $508 \mathrm{MF}$, Kemppinen A, et al. Analysis of immune-related loci identifies 48 new susceptibility variants for 509 multiple sclerosis. Nat Genet. 2013;45(11):1353-60.

$510 \quad 7 . \quad$ International Multiple Sclerosis Genetics C. Multiple sclerosis genomic map implicates 511 peripheral immune cells and microglia in susceptibility. Science. 2019;365(6460).

$512 \quad$ 8. Chitnis T. The role of CD4 T cells in the pathogenesis of multiple sclerosis. Int Rev Neurobiol. 513 2007;79:43-72.

5149 9. Baecher-Allan C, Kaskow BJ, Weiner HL. Multiple Sclerosis: Mechanisms and Immunotherapy. 515 Neuron. 2018;97(4):742-68.

516 10. Ramanathan M, Weinstock-Guttman B, Nguyen LT, Badgett D, Miller C, Patrick K, et al. In vivo 517 gene expression revealed by cDNA arrays: the pattern in relapsing-remitting multiple sclerosis patients 518 compared with normal subjects. J Neuroimmunol. 2001;116(2):213-9.

519 11. Baranzini SE, Mudge J, van Velkinburgh JC, Khankhanian P, Khrebtukova I, Miller NA, et al. 520 Genome, epigenome and RNA sequences of monozygotic twins discordant for multiple sclerosis. 521 Nature. 2010;464(7293):1351-6.

522 12. Gandhi R, Laroni A, Weiner HL. Role of the innate immune system in the pathogenesis of multiple sclerosis. J Neuroimmunol. 2010;221(1-2):7-14.

524 13. Achiron A, Feldman A, Magalashvili D, Dolev M, Gurevich M. Suppressed RNA-polymerase 1 pathway is associated with benign multiple sclerosis. PLoS One. 2012;7(10):e46871.

14. Graves MC, Benton M, Lea RA, Boyle M, Tajouri L, Macartney-Coxson D, et al. Methylation differences at the HLA-DRB1 locus in CD4+ T-Cells are associated with multiple sclerosis. Mult Scler. 2014;20(8):1033-41.

529 15. Parnell GP, Gatt PN, Krupa M, Nickles D, McKay FC, Schibeci SD, et al. The autoimmune 530 disease-associated transcription factors EOMES and TBX21 are dysregulated in multiple sclerosis and 531 define a molecular subtype of disease. Clin Immunol. 2014;151(1):16-24. 
532

533

534

535

536

537

538

539

540

541

542

543

544

545

546

547

548

549

550

551

552

553

554

555

556

557

558

559

560

561

562

563

564

565

566

567

568

569

16. Bos SD, Page CM, Andreassen BK, Elboudwarej E, Gustavsen MW, Briggs F, et al. Genomewide DNA methylation profiles indicate CD8+ T cell hypermethylation in multiple sclerosis. PLoS One. 2015;10(3):e0117403.

17. Maltby VE, Graves MC, Lea RA, Benton MC, Sanders KA, Tajouri L, et al. Genome-wide DNA methylation profiling of CD8+ T cells shows a distinct epigenetic signature to $C D 4+T$ cells in multiple sclerosis patients. Clin Epigenetics. 2015;7:118.

18. Kular L, Liu Y, Ruhrmann S, Zheleznyakova G, Marabita F, Gomez-Cabrero D, et al. DNA methylation as a mediator of HLA-DRB1*15:01 and a protective variant in multiple sclerosis. Nat Commun. 2018;9(1):2397.

19. Rhead B, Brorson IS, Berge T, Adams C, Quach H, Moen SM, et al. Increased DNA methylation of SLFN12 in CD4+ and CD8+ T cells from multiple sclerosis patients. PLoS One. 2018;13(10):e0206511.

20. Ruhrmann S, Ewing E, Piket E, Kular L, Cetrulo Lorenzi JC, Fernandes SJ, et al. Hypermethylation of MIR21 in CD4+ T cells from patients with relapsing-remitting multiple sclerosis associates with lower miRNA-21 levels and concomitant up-regulation of its target genes. Mult Scler. 2018;24(10):1288-300.

21. Brorson IS, Eriksson A, Leikfoss IS, Celius EG, Berg-Hansen P, Barcellos LF, et al. No differential gene expression for CD4(+) T cells of MS patients and healthy controls. Mult Scler J Exp Transl Clin. 2019;5(2):2055217319856903.

22. Brorson IS, Eriksson AM, Leikfoss IS, Vitelli V, Celius EG, Luders T, et al. CD8(+) T cell gene expression analysis identifies differentially expressed genes between multiple sclerosis patients and healthy controls. Mult Scler J Exp TransI Clin. 2020;6(4):2055217320978511.

23. Kim K, Probstel AK, Baumann R, Dyckow J, Landefeld J, Kogl E, et al. Cell type-specific transcriptomics identifies neddylation as a novel therapeutic target in multiple sclerosis. Brain. 2021;144(2):450-61.

24. Payne SH. The utility of protein and mRNA correlation. Trends Biochem Sci. 2015;40(1):1-3.

25. Schwanhausser B, Wolf J, Selbach M, Busse D. Synthesis and degradation jointly determine the responsiveness of the cellular proteome. Bioessays. 2013;35(7):597-601.

26. Berge T, Eriksson A, Brorson IS, Hogestol EA, Berg-Hansen P, Doskeland A, et al. Quantitative proteomic analyses of $\mathrm{CD} 4(+)$ and $\mathrm{CD} 8(+)$ T cells reveal differentially expressed proteins in multiple sclerosis patients and healthy controls. Clin Proteomics. 2019;16:19.

27. Hellberg S, Eklund D, Gawel DR, Kopsen M, Zhang H, Nestor CE, et al. Dynamic Response Genes in CD4+ T Cells Reveal a Network of Interactive Proteins that Classifies Disease Activity in Multiple Sclerosis. Cell Rep. 2016;16(11):2928-39.

28. Quinn EM, Coleman C, Molloy B, Dominguez Castro P, Cormican P, Trimble V, et al. Transcriptome Analysis of CD4+ T Cells in Coeliac Disease Reveals Imprint of BACH2 and IFNgamma Regulation. PLoS One. 2015;10(10):e0140049.

29. Polman $\mathrm{CH}$, Reingold SC, Banwell B, Clanet M, Cohen JA, Filippi M, et al. Diagnostic criteria for multiple sclerosis: 2010 revisions to the McDonald criteria. Ann Neurol. 2011;69(2):292-302. 
570 30. Hughes CS, Moggridge S, Muller T, Sorensen PH, Morin GB, Krijgsveld J. Single-pot, solid571 phase-enhanced sample preparation for proteomics experiments. Nat Protoc. 2019;14(1):68-85.

572 31. Liebmann M, Hucke S, Koch K, Eschborn M, Ghelman J, Chasan Al, et al. Nur77 serves as a 573 molecular brake of the metabolic switch during $T$ cell activation to restrict autoimmunity. Proc Natl 574 Acad Sci U S A. 2018;115(34):E8017-E26.

575 32. Kim K, Probstel AK, Baumann R, Dyckow J, Landefeld J, Kogl E, et al. Cell type-specific 576 transcriptomics identifies neddylation as a novel therapeutic target in multiple sclerosis. Brain. 2020.

577 33. Su X, Lin Z, Chen W, Jiang H, Zhang S, Lin H. Chemogenomic approach identified yeast 578 YLR143W as diphthamide synthetase. Proc Natl Acad Sci U S A. 2012;109(49):19983-7.

579 34. Pavitt GD, Proud CG. Protein synthesis and its control in neuronal cells with a focus on 580 vanishing white matter disease. Biochem Soc Trans. 2009;37(Pt 6):1298-310.

581 35. Shimono $\mathrm{Y}$, Murakami $\mathrm{H}$, Hasegawa $\mathrm{Y}$, Takahashi $\mathrm{M}$. RET finger protein is a transcriptional repressor and interacts with enhancer of polycomb that has dual transcriptional functions. J Biol Chem. 2000;275(50):39411-9.

584 36. Ellis JH, Ashman C, Burden MN, Kilpatrick KE, Morse MA, Hamblin PA. GRID: a novel Grb-2related adapter protein that interacts with the activated $T$ cell costimulatory receptor CD28. J Immunol. 2000;164(11):5805-14.

587 37. Vanshylla K, Bartsch C, Hitzing C, Krumpelmann L, Wienands J, Engels N. Grb2 and GRAP 588 connect the B cell antigen receptor to Erk MAP kinase activation in human B cells. Sci Rep. 589 2018;8(1):4244.

590 38. Feng GS, Ouyang YB, Hu DP, Shi ZQ, Gentz R, Ni J. Grap is a novel SH3-SH2-SH3 adaptor protein 591 that couples tyrosine kinases to the Ras pathway. J Biol Chem. 1996;271(21):12129-32.

39. Shen R, Ouyang YB, Qu CK, Alonso A, Sperzel L, Mustelin T, et al. Grap negatively regulates Tcell receptor-elicited lymphocyte proliferation and interleukin-2 induction. Mol Cell Biol. 2002;22(10):3230-6.

40. Couturier N, Bucciarelli F, Nurtdinov RN, Debouverie M, Lebrun-Frenay C, Defer G, et al. Tyrosine kinase 2 variant influences $\mathrm{T}$ lymphocyte polarization and multiple sclerosis susceptibility. Brain. 2011;134(Pt 3):693-703.

598 41. Wu R, He Q, Chen $\mathrm{H}, \mathrm{Xu} \mathrm{M}$, Zhao N, Xiao $\mathrm{Y}$, et al. MicroRNA-448 promotes multiple sclerosis 599 development through induction of Th17 response through targeting protein tyrosine phosphatase 600 non-receptor type 2 (PTPN2). Biochem Biophys Res Commun. 2017;486(3):759-66.

601 42. Traboulsee AL, Sadovnick AD, Encarnacion M, Bernales CQ, Yee IM, Criscuoli MG, et al. 602 Common genetic etiology between "multiple sclerosis-like" single-gene disorders and familial multiple 603 sclerosis. Hum Genet. 2017;136(6):705-14.

604 43. Khatami M, Heidari MM, Mansouri R, Mousavi F. The POLG Polyglutamine Tract Variants in 605 Iranian Patients with Multiple Sclerosis. Iran J Child Neurol. 2015;9(1):37-41.

606 


\section{Figures}

A
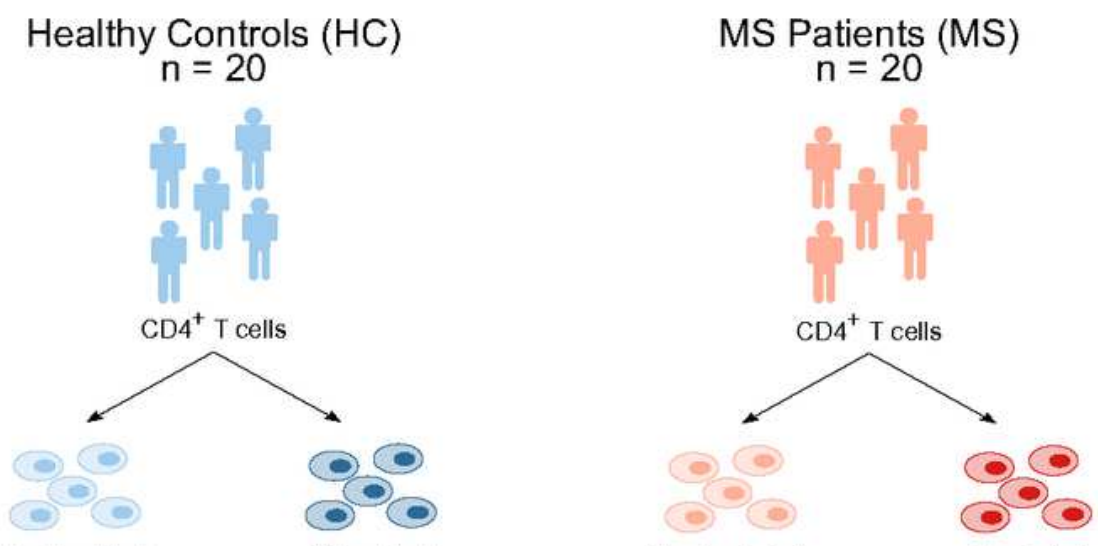

Unstimulated

Stimulated

Unstimulated

Stimulated

5,704

quantified proteins

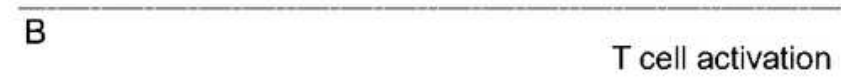

Unstimulated

T cell activation

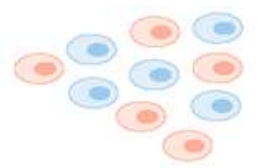

differrentially expressed proteins

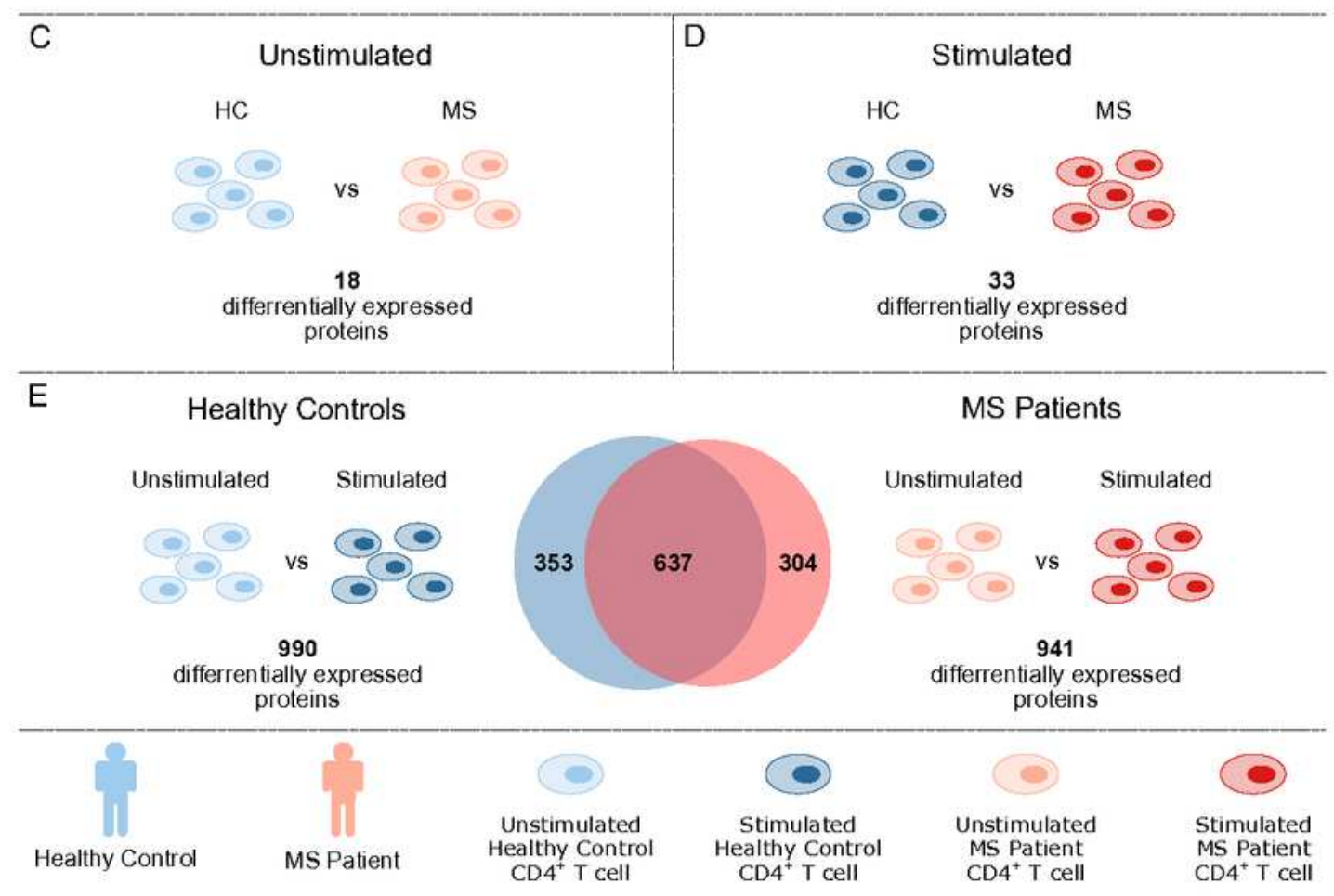

Figure 1

An overview of the study. Study design (A). Differentially expressed proteins between unstimulated and stimulated CD4+ T cells (B). Differentially expressed proteins between HC and MS in unstimulated CD4+ T cells (C) and in stimulated CD4+ T cells (D). Proteins that change in abundance upon CD4+ T cell 
activation of samples from MS and $\mathrm{HC}(\mathrm{E})$. The Venn diagram displays the number of proteins that were differentially expressed between unstimulated and stimulated CD4+ T cells from HCs (blue) and MS patients (red).

A

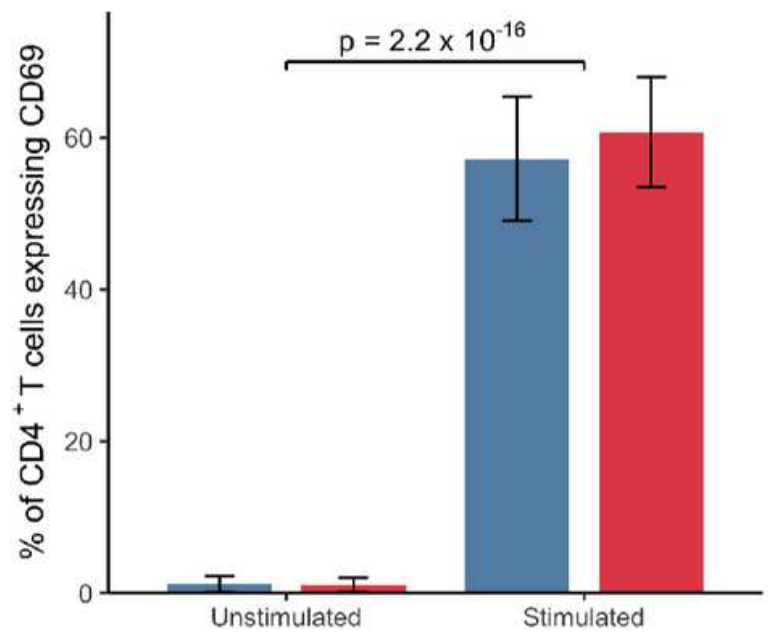

B

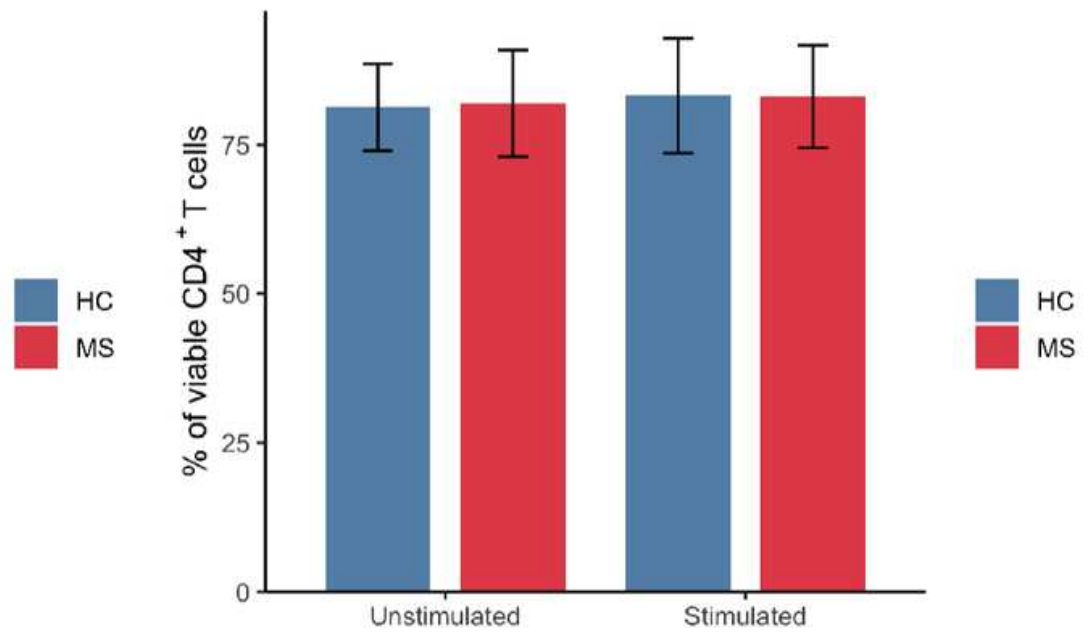

Figure 2

Flow cytometry characterization of CD4+ T cells from MS patients (MS) and healthy controls $(H C)$. Proportions of (A) CD69+ and (B) viable CD4+ T cells in unstimulated and stimulated (anti-CD3/CD28 antibody stimulation) samples. Mean and standard deviation are shown, Mann Whitney $U$ test was used for paired comparisons, only significant $p$-values $(p>0.05)$ are depicted in the figure. MS $=$ multiple sclerosis, $\mathrm{HC}=$ healthy controls.

\section{A Unstimulated $\mathrm{CD}^{+} \mathrm{T}$ cells}

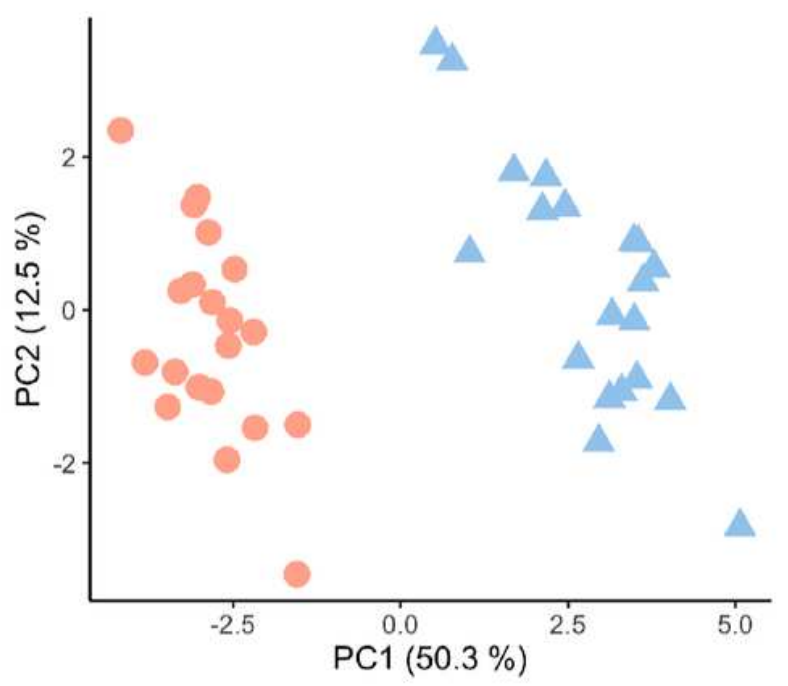

B Stimulated $\mathrm{CD}^{+} \mathrm{T}$ cells

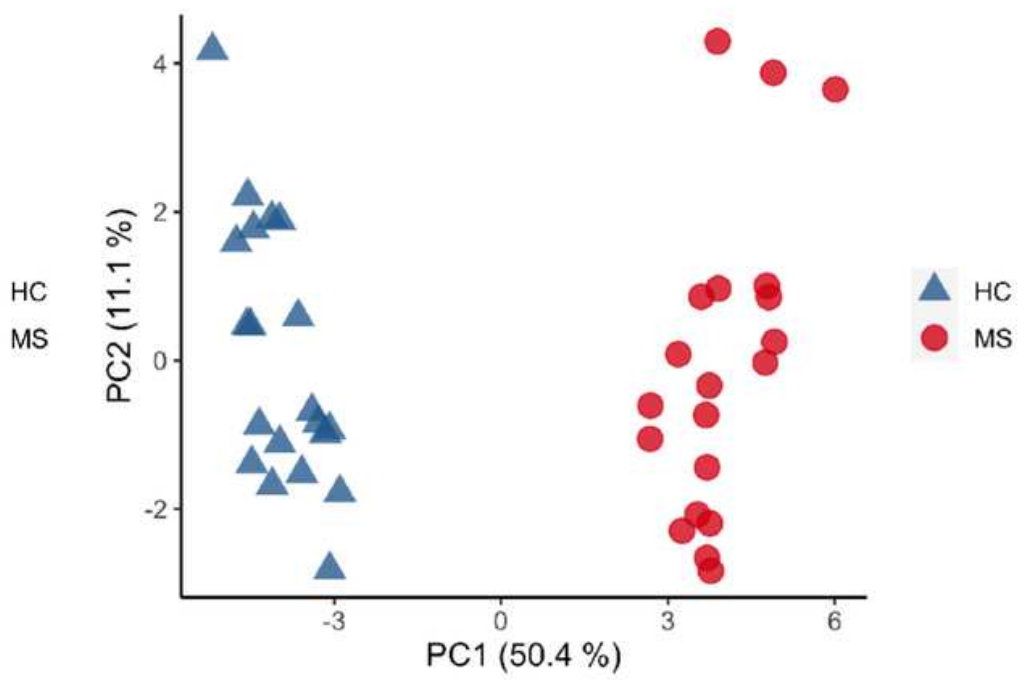

\section{Figure 3}


Principal component analysis (PCA) of differentially expressed proteins. Scores of the first (PC1) and second (PC2) principal components obtained by PCA of proteins significantly different in abundance ( $\mathrm{p} \leq$ 0.05 ) between MS patients (MS; red circles) and HCs (HC; blue triangles) in (A) unstimulated and (B) stimulated CD4+ T cells.

A

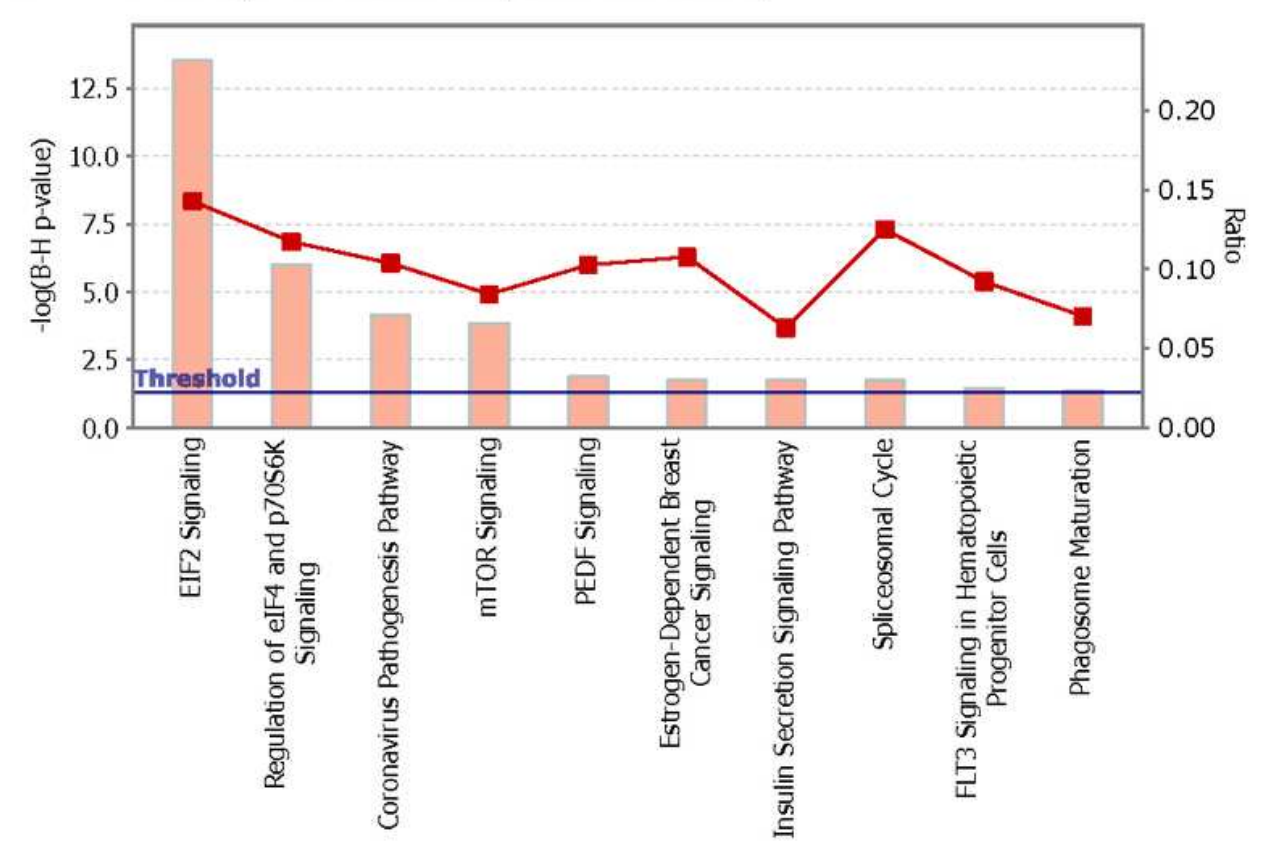

B Pathways enriched in $\mathrm{HC}$ exclusively

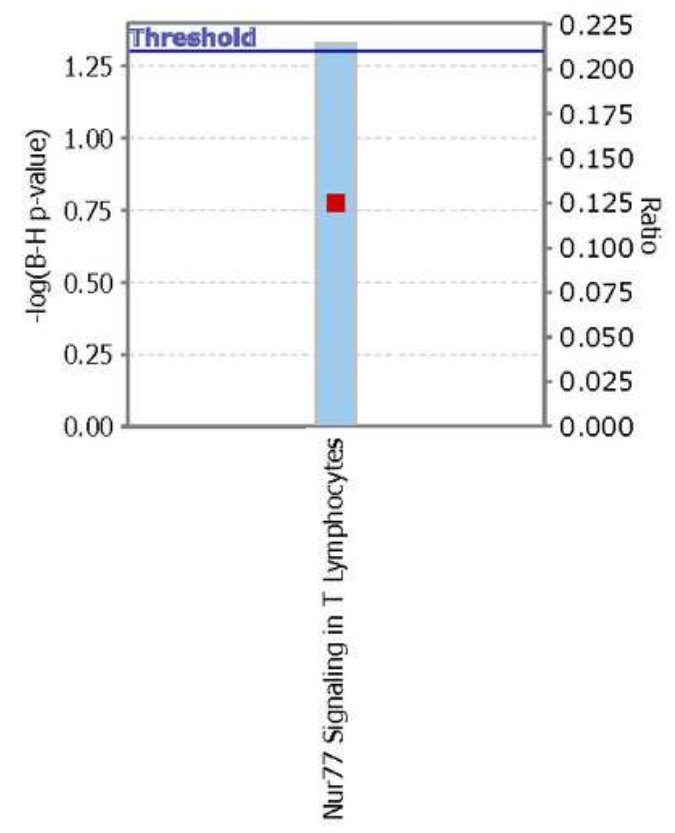

\section{Figure 4}

Biological pathways enriched upon $\mathrm{CD} 4+\mathrm{T}$ cell activation. The graphs display the biological pathways enriched among proteins that are differentially expressed between unstimulated and stimulated CD4+ $T$ cells exclusively in (A) MS patients (pink) or (B) HCs (light blue) after Benjamini-Hochberg (B-H) multiple testing correction ( $p$-values seen on left y axis, blue line is marking the threshold level for significance). The red line with squares represents the ratio of the number of proteins in the data set of differentially expressed proteins divided by the number of proteins in the reference data set for that specific pathway (right y axis).

\section{Supplementary Files}

This is a list of supplementary files associated with this preprint. Click to download.

- TablesCappellettietal.pdf 